\title{
Sciatic Nerve Cut and Repair Using Fibrin Glue in Adult Mice
}

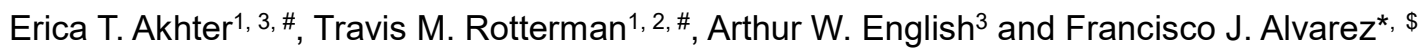

\author{
1Department of Physiology, Emory University, Atlanta, USA; ${ }^{2}$ School of Biological Sciences, Georgia \\ Institute of Technology, Atlanta, USA; ${ }^{3}$ Department of Cell Biology, Emory University, Atlanta, USA; \\ \$Current address: Department of Physiology, Emory University, Atlanta, GA 30322, USA \\ *For correspondence: Francisco.j.alvarez@emory.edu \\ \#Contributed equally to this work
}

\begin{abstract}
[Abstract] Peripheral nerve injury (PNI) is an excellent model for studying neural responses to injury and elucidating the mechanisms that can facilitate axon regeneration. As such, several animal models have been employed to study regenerative mechanisms after PNI, including Aplysia, zebrafish, rabbits, cats and rodents. This protocol describes how to perform a sciatic nerve injury and repair in mice, one of the most frequently used models to study mechanisms that facilitate recovery after PNI, and that takes advantage of the availability of many genetic models. In this protocol, we describe a method for using fibrin glue to secure the proximal and distal stumps of an injured nerve in close alignment. This method facilitates the alignment of nerve stumps, which aids in regeneration of both sensory and motor axons and allows successful reconnection with peripheral targets.
\end{abstract}

Keywords: Nerve, Injury, Regeneration, Sciatic, Repair, Axotomy, Bridging gaps, Mice

[Background] Peripheral nerve injuries (PNIs) are an important clinical problem and recovery outcomes are generally poor (Jensen et al., 2001; Höke and Brushart, 2010). Additionally, treatments for these injuries have not much changed in the last 100 years (Mundie, 1920; Taylor et al., 2008). As such, models of PNI have become important to understand potential barriers to regeneration and treatments for enhancing it. While in humans and larger mammals, such as cats, suturing through the epineurium is a common mechanism of repair (Lundborg, 2002; Grinsell and Keating, 2014; Raslan et al., 2014), this treatment is not reliably performed in mice. First, the diameter of the sciatic nerve is less than $2 \mathrm{~mm}$ making the placement of epineurial sutures technically challenging. Second, in comparison to nerves in larger animals, axons in mouse nerves are more likely to exude from cut nerves or "mushroom out" when the epineurium is penetrated during placement of sutures, making clean repair of the cut nerves difficult. Finally, placement of sutures in small rodents can interfere with the blood supply of regenerating nerves. One method that can overcome these challenges is the use of fibrin glue, a tool that was first utilized in human patients (Egloff and Narkas, 1983; MacGillivray, 2003). The implementation of fibrin glue in experimental animals (as models to assess its benefits for human nerve repair) also has a long history; decades ago it was found to be as effective as epineurial sutures for repair of the rat sciatic nerve (Nishihira, 1988). We describe a method to repair a mouse sciatic nerve injury that facilitates nerve regeneration in experimental situations. First, we use a fibrin glue formula, derived from human and bovine blood components, that was previously found to enhance repair in 
central nervous system axon tracts (Guest et al., 1997). Second, we secure the nerve by adhering it to a silastic mat prior to transection. This ensures a minimal gap between the proximal and distal nerve stumps and enhanced stability, without having to leave a piece of the epineurium intact, which is a technically difficult approach and can result in leaving some axons uninjured. The improved coaptation and increased stability diminishes the incidence of dehiscence reported without suture stabilization (Cruz et al., 1986). While the components of fibrin glue may interact with the immune system (Schoenecker et al., 2001; Mosesson, 2005; Kelsh et al., 2014), this strategy has been used extensively in both rats and mice with great success. Comprehensive comparisons of nerve repair efficiency using fibrin glues compared to epineurial sutures have been recently published (Adel et al., 2017; Koulaxouzidis et al., 2017, Wang et al., 2018). In our hands, the protocol described facilitates sciatic nerve reformation in mice by two months following injury as quantified by complete motor muscle reinnervation assessed by re-occupancy of neuromuscular junctions and electromyography with an almost 100\% success rate (Wilhelm et al., 2012; Rotterman et al., 2019). As a further advantage, drugs or other compounds can be added to the glue to test how different factors administered peripherally (and directly on the nerve) may impact regeneration (Wilhelm et al., 2012). These nerve repair surgeries are all done in adult (3+ month) C57Blk/6 mice, but can easily be transferred to other species and ages.

\section{Materials and Reagents}

1. Cohen-Vannas Scissors (Fine Science Tools, catalog number: 15000-01)

2. Sterile Poly-lined drape (Medline, catalog number: NON21001)

3. \#3 and \#5 Forceps

4. Nose cone

5. (Optional) Gelfoam ${ }^{\circledR}$ (Pfizer, catalog number: 1575-9039605)

6. $10 \mu \mathrm{l}$ pipette (Gilson, Pipetman, catalog number: F144802)

7. SILASTIC ${ }^{\circledR}$ film (Dow Corning, catalog number: 501-1). This material is medical grade silicone rubber sheets specifically designed for implantation

8. Surgical tape (Dynarex, Surgical Tape Cloth, catalog number: 3562)

9. Sterile Stainless Steel Surgical Blades (Integra Militex Kai, catalog number: 4-311)

10. $10 \mu$ pipette tips (Lab Depot, MicroPette Universal Tips, catalog number: 750001)

11. Sterile gauze (Fisherbrand, catalog number: 22-362-178)

12. $1 / 2 \mathrm{ml}$ Monoject Insulin Syringe, $29 \mathrm{G} \mathrm{x} \mathrm{1/2"} \mathrm{(Covidien,} \mathrm{catalog} \mathrm{number:} \mathrm{8881600350)}$

13. Adult C57Blk/6 mice ( 3 months + old, alternative animal strains can be used as well)

14. *Thrombin (25 Units/ml in $45 \mathrm{mmol} / \mathrm{L} \mathrm{CaCl}$ ) (MP BioChemicals, LLC, catalog number: 154163)

15. *Fibrinogen $(100 \mathrm{mg} / \mathrm{ml})$ (Sigma, catalog number: F3879)

16. *Fibronectin (8 mg/ml) (Sigma, catalog number: F1141)

17. Buprenorphine hydrochloride (Rechitt Benckiser Healthcare (UK) Ltd., Buprenex ${ }^{\circledR}$ Injection, catalog number: RXBUPRENOR5 via shopmedvet.com) 
18. Lubricant Eye Ointment (Major Pharmaceuticals, LubriFesh ${ }^{\mathrm{TM}}$ P.M., catalog number: NDC 0904-6488-38)

19. 5-0 Polydioxanone (KeeBomed, $20 \mathrm{~mm}$ reverse cutting Polydioxanone suture)

20. Isoflurane, USP (Piramal Critical Care, material number: 400648037)

21. $70 \%$ Isopropyl Alcohol (Medline, Medium Alcohol Prep Pads, catalog number: MDS090735)

22. lodine (Medline, Medium Prep Pads Povidone-lodine, USP, catalog number: NDC 53329-944-30)

23. Hair Remover Lotion (Nair, Church and Dwight Co, catalog number: 70501345)

*Note: Aliquots of these components may be made up to 6 months in advance and stored separately at $-20{ }^{\circ} \mathrm{C}$ until just prior to use. They must be mixed immediately prior to use as they begin to coagulate immediately.

\section{Equipment}

1. Retractors (Fine Science Tools, Mini-Goldstein, catalog number: 17002-02)

2. Electric hair clippers (Wahl, catalog number: 99160)

3. Dissecting microscope (Olympus Tokyo, catalog number: 222855)

4. Warm water pump (Gaymar, catalog number: TP-500)

5. 2 Heating pads (one for surgery and one for recovery) (Adroit Medical Systems, catalog number: V016)

6. Oxygen concentrator (SupraVet, Pureline oxygen concentrator, catalog number: OC6000)

7. Isoflurane vaporizer (VetEquip, Vaporizer- Funnel-Fill, catalog number: 911103)

8. Induction chamber, hoses, filters and connectors (VetEquip, Dual Procedure Circuit, catalog number: 921400)

9. Cotton Tipped Applicators (MediChoice, catalog number: WOD1002)

10. Dumont \#3c (Fine Science Tools, catalog number: 11231-20)

11. Secure Scalpel Handle \#3 (Fine Science Tools)

\section{Procedure}

A. Surgical preparation

1. Sterilize all equipment and place drape over circulating water heated pad to maintain sterile field and keep animal warm through the duration of the surgery. Water is circulated by a water pump with temperature controls.

2. Thaw aliquots of thrombin, fibrinogen, and fibronectin to room temperature. Fibrinogen will begin to coagulate independently if left at room temperature for too long, so ensure there is minimal time between thawing and surgery.

Note: We have successfully used these up to $6 h$ after thawing.

3. Weigh the animal and prepare a fresh dose of buprenorphine (0.05-0.1 mg/kg) administered 
intraperitoneally prior to anesthetic induction.

4. Induce anesthesia by placing the animal in the isoflurane box (approximately $4 \%$ isoflurane in $100 \%$ oxygen) for approximately $4 \mathrm{~min}$.

5. The mouse is then placed on a nose cone, in a separate area to prep the leg.

6. Remove hair on the dorsal hindlimb (ankle to hip) using electric clippers and/or Nair. Apply eye ointment with a sterile cotton applicator.

7. Wipe the surgical area with alcohol and iodine wipes three times in succession (rubbing from middle to edges of limb).

8. Confirm a surgical plane of anesthesia by applying pressure to hindpaw with forceps (toe pinch). Wait to proceed until there is no response.

B. Surgery (the entire procedure is performed under a stereo dissecting microscope)

1. The animal is then moved into the surgical field and placed on a nose-cone to supply isoflurane anesthesia for the remainder of the surgery. Secure hindlimb in extended position with surgical tape (Figure 1). In this example we are using the left leg, however the same procedure can be performed on the right leg or in both.
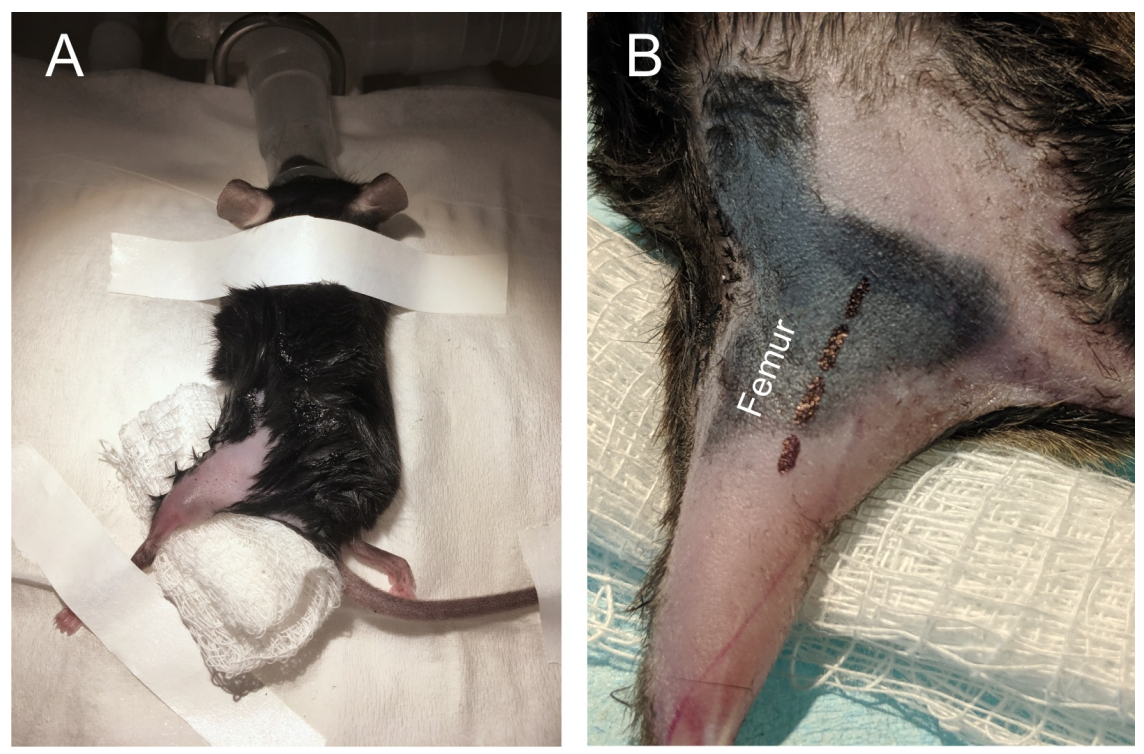

Figure 1. Mouse orientation and incision site. A. The mouse was anesthetized via isoflurane and secured with leg extended. B. Close-up of leg in extended position. Black dashed line outlines the location of the sciatic nerve (located medial to the femur).

2. Palpate the skin to identify the femur, then move $2 \mathrm{~mm}$ medial and make a small incision $(<2$ $\mathrm{cm}$ ) in the skin with a fine scalpel (Figure 2). 


\section{biö-protocol

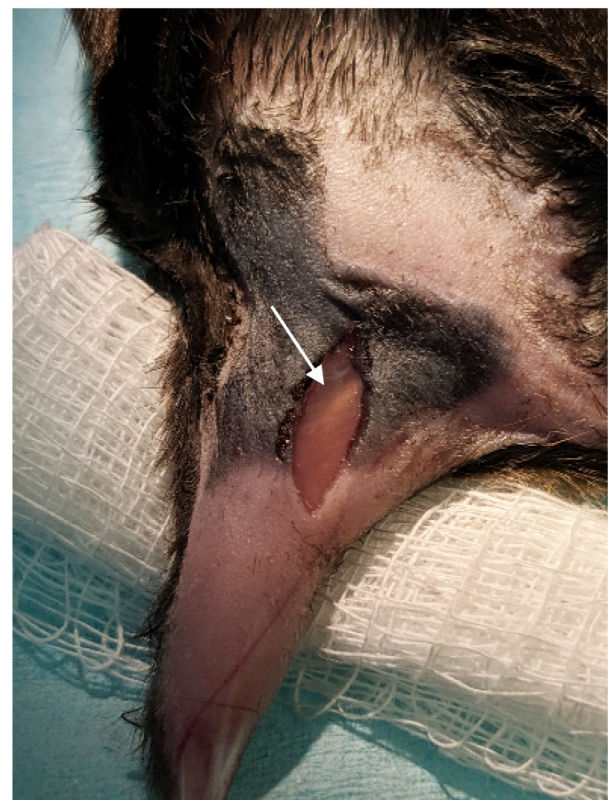

Figure 2. Incision. A small incision was made through the skin just over the sciatic nerve. The sciatic nerve can be seen through the biceps femoris muscle (arrow). The sciatic is just lateral the femur bone. Medial to the nerve there is a blood vessel that runs parallel. Once the biceps muscle is retracted the sciatic nerve will be obvious as it is the largest nerve in the hind limb.

3. Blunt dissect muscle (biceps femoris) at mid-thigh using extra fine Bonn scissors (FST catalog number 14084-08), exposing the sciatic nerve. A retractor may be used to ensure adequate visibility, but is not necessary during a typical surgery. Carefully isolate the nerve from connective tissue (Figure 3). The nerve is carefully isolated using a pair of \#5 forceps.

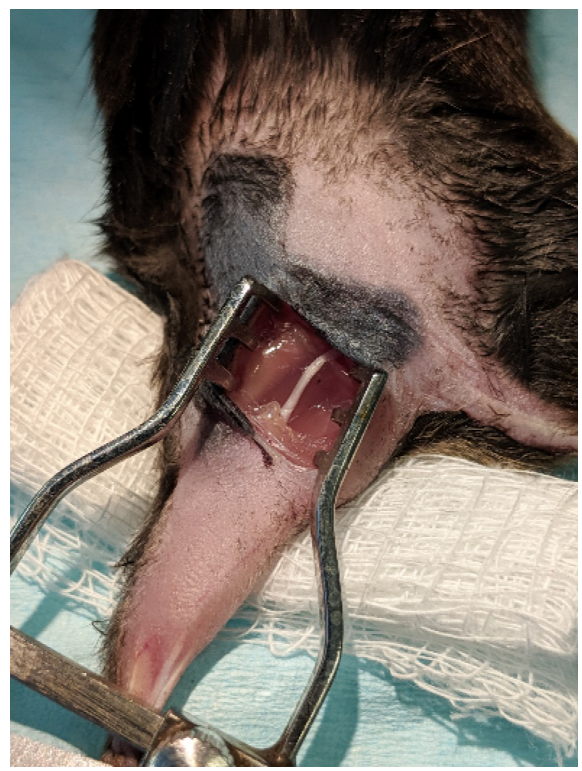

Figure 3. Exposure of the sciatic nerve. The biceps femoris was blunt dissected and a pair of retractors inserted to separate skin and muscle over the sciatic nerve. 


\section{bĭo-protocol

4. Place a small ( 4 $\mathrm{mm} \times 4 \mathrm{~mm}$ square) of SILASTIC film below the sciatic nerve (Figure 4).

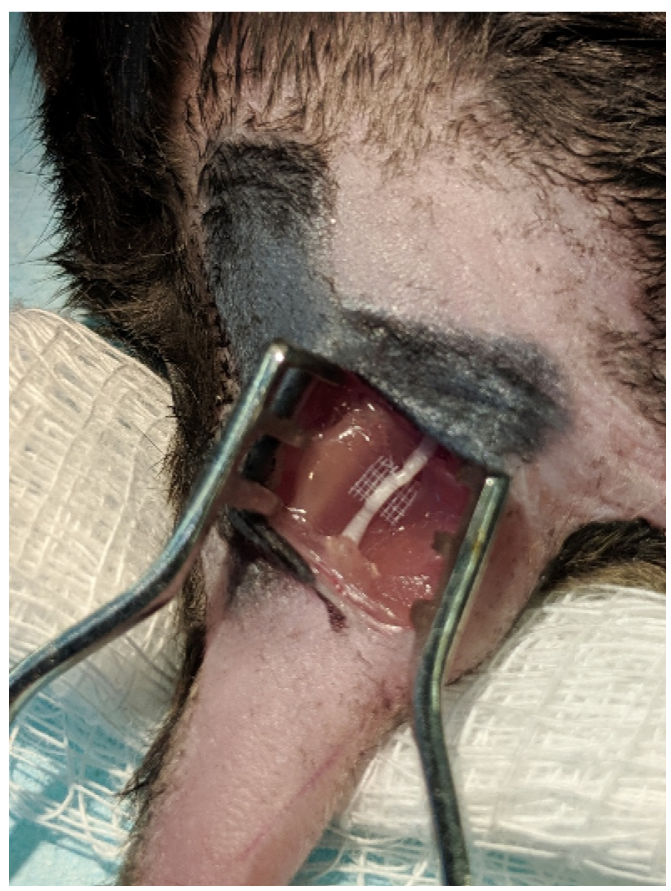

Figure 4. SILASTIC platform. A small piece of SILASTIC matting was placed under the sciatic nerve.

5. Cut sciatic nerve with sharp microscissors, leaving the film intact (Figure 5).

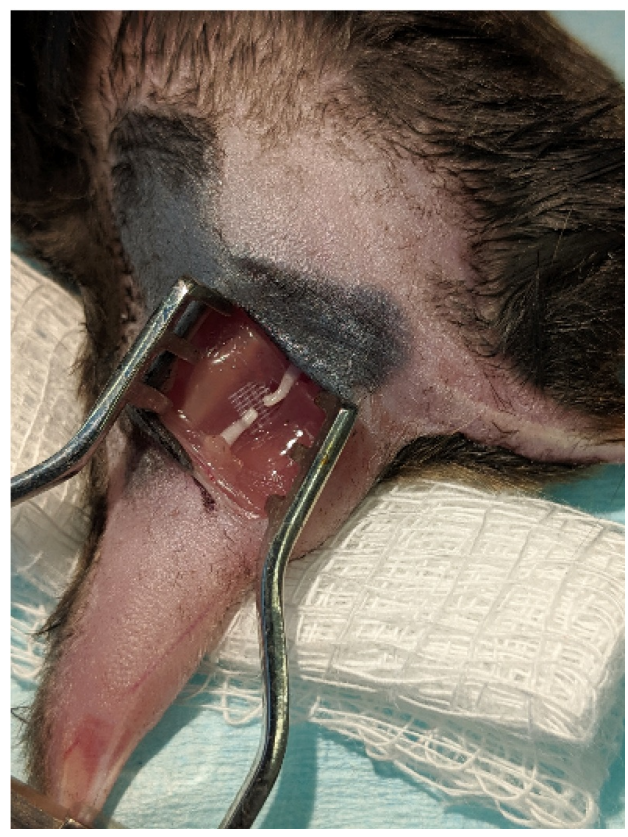

Figure 5. Nerve Injury. The sciatic nerve was completely transected with small surgical scissors, leaving both stumps on the SILASTIC mat. 
6. Quickly mix fibrinogen/fibronectin/thrombin in a 1:1:2 ratio on a smooth, sterile surface, using fresh tips each time. We recommend mixing in this order for consistency.

7. Place approximately $10 \mu \mathrm{l}$ mixture quickly on sciatic nerve over SILASTIC film (Figure 6). Wait until glue becomes tacky (usually $\sim 2 \mathrm{~min}$ ) prior to next steps.

Note: Here we placed $20 \mu \mathrm{l}$ for demonstration purposes, however $10 \mu \mathrm{l}$ is sufficient for securing the nerve stumps together. If the nerve stumps are not both covered in glue then more may need to be added, though this is unlikely.

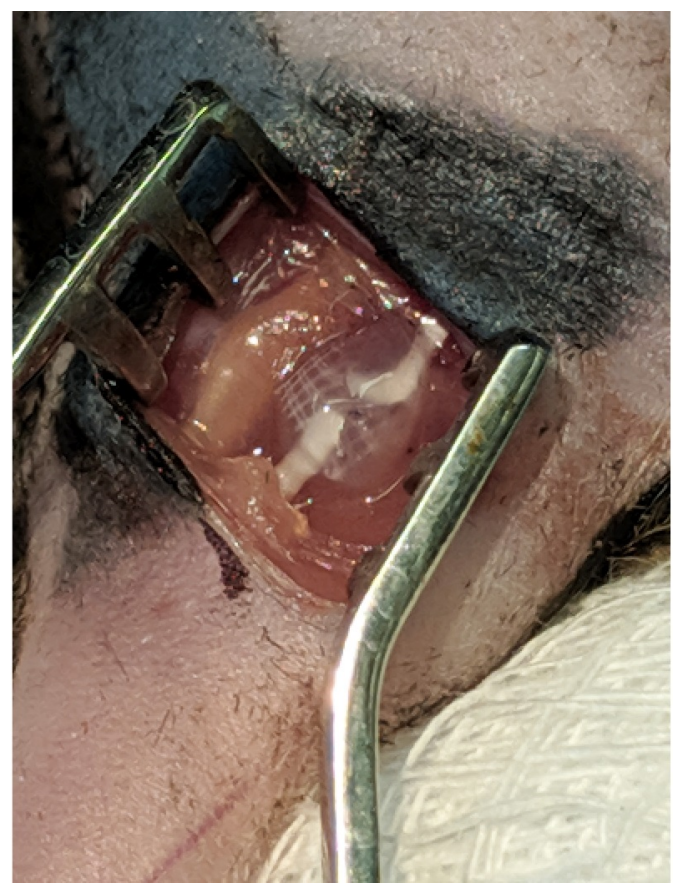

Figure 6. Nerve repair. The sciatic nerve was then glued back together using a cocktail containing fibrinogen $(2 \mathrm{mg} / \mathrm{ml})$, fibronectin $(25 \mathrm{mg} / \mathrm{ml})$, and thrombin (12.5 Units $/ \mathrm{ml})$.

8. Ensure there is no excess bleeding and close the wound in layers, with loose sutures through the belly of the muscle and tight sutures through the skin (Figures 7 and 8). 


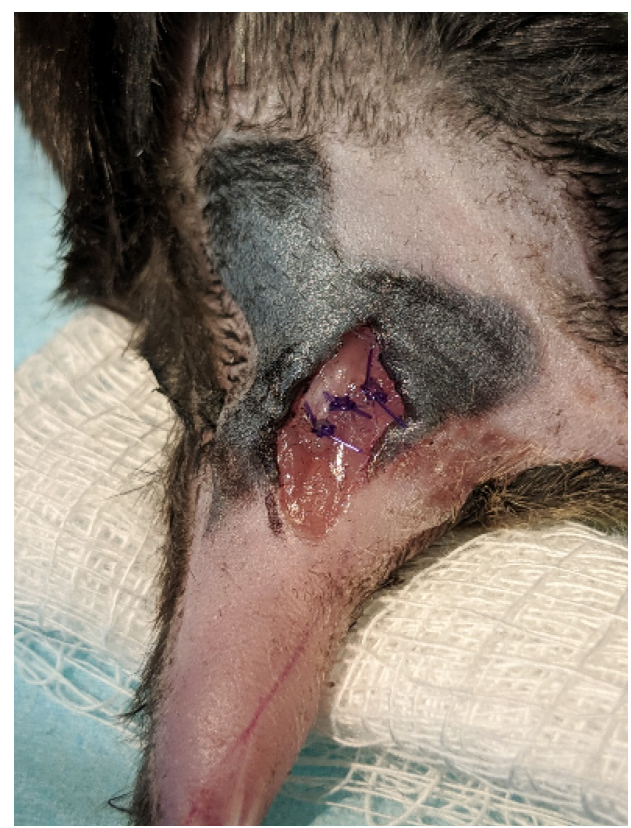

Figure 7. Muscle closure. The biceps femoris muscle is sutured back together using absorbable suture.

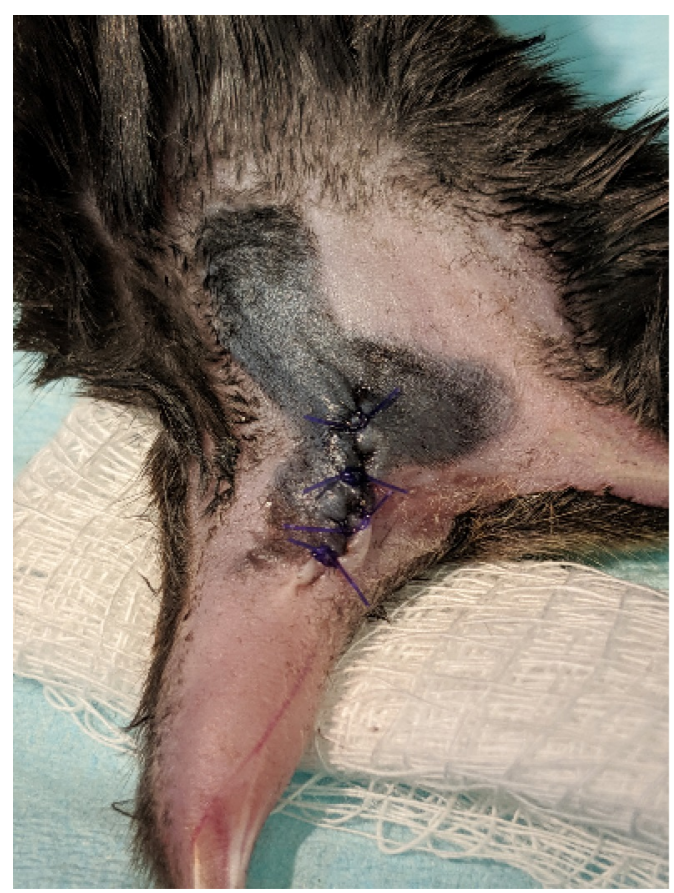

Figure 8. Skin closure. The skin is realigned and sutured back together using absorbable suture.

Note: One can also use wound clips for the step.

\section{Surgery Completion}

Remove the mouse from isoflurane and keep on heating pad until mouse becomes conscious and moves freely. 
Following this procedure the nerve is repaired and there is continuity between proximal and distal stumps (Figure 9). Axons successfully traverse the gap between the cut stumps and ultimately reconnect and reinnervate muscle within an 8-week period (Willherm et al., 2012; Rotterman et al., 2019).

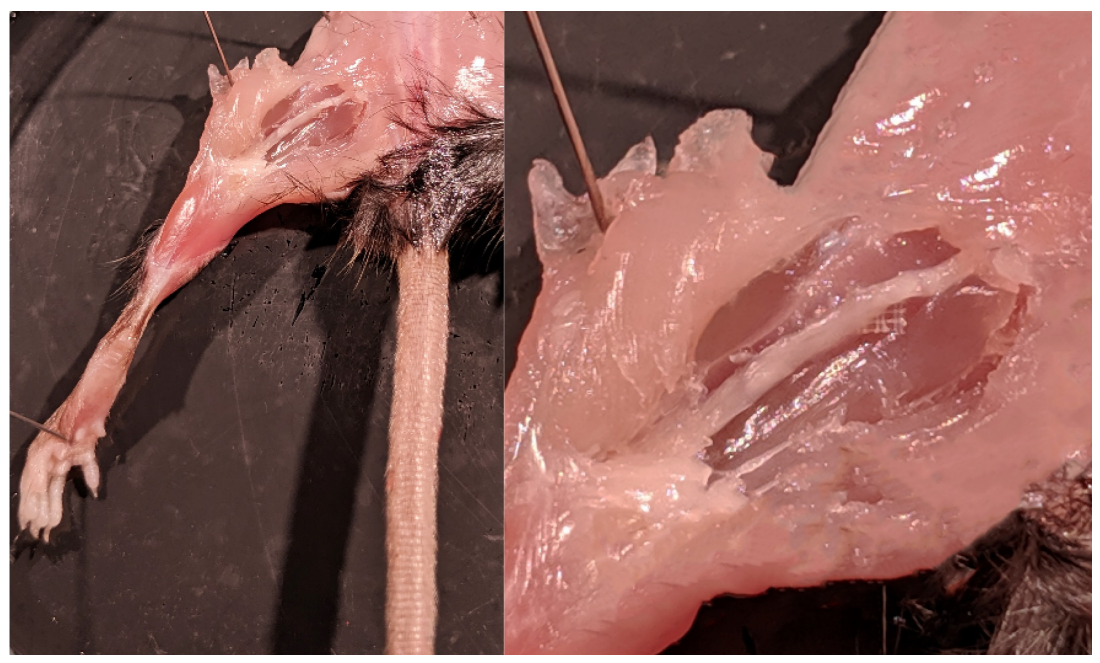

Figure 9. Nerve repair 8 weeks post injury. These images show a successful nerve repair 8 weeks after injury in a mouse perfused with paraformaldehyde fixative. The nerve was completely reformed at this time point and the axons re-innervated muscles in the lower hindlimb.

\section{$\underline{\text { Notes }}$}

1. Fibronectin, fibrinogen and thrombin mixtures coagulate quickly and should be prepared fresh each day prior to surgery. If making large aliquots for multiple surgeries ensure that fresh pipette tips are used to extract solution each time as even contact mixing may cause premature coagulation of the aliquots.

2. If excessive bleeding occurs, stop bleeding with Gelfoam ${ }^{\circledR}$ (Pfizer) prior to continuation with surgery. The repair area must be relatively dry to ensure proper coagulation of fibrin glue.

3. Additional glue may be used to secure the nerve further after surgery if necessary. The fibrin glue needs to stabilize the two nerve stumps, if any of the two nerve stumps is not perfectly secure or covered more fibrin glue can be added.

4. Sharp scissors are imperative for the nerve cut to ensue minimal feathering of nerve ends and no gap between proximal and distal nerve stumps.

5. Nerve repairs are initially fragile after surgery. Be careful when moving mice while anesthetized not to disturb the leg. Connective tissue quickly holds the repair in place in the days following surgery.

6. As a control, sham surgeries should be conducted by exposing the nerve and freeing it from connective tissue. Placing of SILASTIC mat without fibrin glue and/or nerve cut may also serve 
as appropriate shams.

\section{Acknowledgments}

This work was supported by the grants from the National Institute of Neurological Disorders and Stroke Grants P01NS057228 and R56NS099092 to F.J.A., NS057196 to A.W.E. and a Ruth L. Kirschstein National Research Service Award F31NS095528 fellowship to T.M.R. E.T.A was supported by a National Science Foundation Graduate Fellowship DGE-1444932.

This protocol was adapted from the original proposal of using fibrin to repair peripheral nerves in humans (Egloff and Narakas, 1983) and rodents (Nishihira, 1988) and further development of addition a fibrin/fibrinogen/thrombin combination to enhance axon growth as applied to CNS injuries (Guest et al., 1997).

\section{Competing interests}

The corresponding author (F.J.A.) declares that the authors have no financial or non-financial competing interests related to the work described here.

\section{Ethics}

Animal care, procedures, and euthanasia were performed under prior approval by the Institutional Animal Care and Use Committee of the Author's University and in accordance with the NIH Guide for the Care and Use of Laboratory Animals.

\section{References}

1. Adel, M., Abdo Elgamal, D., Bakry, R., Abdelkader, M., Elshazly, M. and Kamel, A. (2017). Suture versus fibrin glue microneural anastomosis of the femoral nerve in sprague dewly rat model. A comparative experimental assessment of the clinical, histological and statistical features. Acta Chir Plast 59(2): 65-71.

2. Cruz, N. I., Debs, N. and Fiol, R. E. (1986). Evaluation of fibrin glue in rat sciatic nerve repairs. Plast Reconstr Surg 78(3): 369-373.

3. Egloff, D. V. and Narakas, A. (1983). Nerve anastomoses with human fibrin. Preliminary clinical report (56 cases). Ann Chir Main 2(2): 101-115.

4. Grinsell, D. and Keating, C. P. (2014). Peripheral nerve reconstruction after injury: a review of clinical and experimental therapies. Biomed Res Int 2014: 698256.

5. Guest, J. D., Hesse, D., Schnell, L., Schwab, M. E., Bunge, M. B. and Bunge, R. P. (1997). Influence of IN-1 antibody and acidic FGF-fibrin glue on the response of injured corticospinal tract axons to human Schwann cell grafts. J Neurosci Res 50(5): 888-905. 
6. Höke, A. and Brushart, T. (2010). Introduction to special issue: Challenges and opportunities for regeneration in the peripheral nervous system. Exp Neurol 223(1): 1-4.

7. Jensen, T. S., Gottrup, H., Sindrup, S. H. and Bach, F. W. (2001). The clinical picture of neuropathic pain. Eur J Pharmacol 429(1-3): 1-11.

8. Kelsh, R., You, R., Horzempa, C., Zheng, M. and McKeown-Longo, P. J. (2014). Regulation of the innate immune response by fibronectin: synergism between the III-1 and EDA domains. PLoS One 9(7): e102974.

9. Koulaxouzidis, G., Reim, G. and Witzel, C. (2015). Fibrin glue repair leads to enhanced axonal elongation during early peripheral nerve regeneration in an in vivo mouse model. Neural Regen Res. 10(7): 1166-1171.

10. Lundborg, G. (2002). Enhancing posttraumatic nerve regeneration. J Peripher Nerv Syst 7(3): 139-140.

11. MacGillivray, T. E. (2003). Fibrin sealants and glues. J Card Surg 18(6): 480-485.

12. Mosesson, M. W. (2005). Fibrinogen and fibrin structure and functions. $J$ Thromb Haemost 3(8): 1894-1904.

13. Mundie, G. S. (1920). Peripheral nerve injuries. Can Med Assoc J 10(3): 294-296.

14. Nishihira, S. (1988). Experimental study of motor nerve repair: comparison of nylon suture and two component fibrin adhesive techniques. Nihon Jibiinkoka Gakkai Kaiho 91(8): 1208-1212.

15. Raslan, A., Ernst, P., Werle, M., Thieme, H., Szameit, K., Finkensieper, M., Guntinas-Lichius, O. and Irintchev, A. (2014). Reduced cholinergic and glutamatergic synaptic input to regenerated motoneurons after facial nerve repair in rats: potential implications for recovery of motor function. Brain Struct Funct 219(3): 891-909.

16. Rotterman, T. M., Akhter, E. T., Lane, A. R., MacPherson, K. P., Garcia, V. V., Tansey, M. G. and Alvarez, F. J. (2019). Spinal motor circuit synaptic plasticity after peripheral nerve injury depends on microglia activation and a CCR2 mechanism. J Neurosci 39(18): 3412-3433.

17. Schoenecker, J. G., Johnson, R. K., Lesher, A. P., Day, J. D., Love, S. D., Hoffman, M. R., Ortel, T. L., Parker, W. and Lawson, J. H. (2001). Exposure of mice to topical bovine thrombin induces systemic autoimmunity. Am J Pathol 159(5): 1957-1969.

18. Taylor, C. A., Braza, D., Rice, J. B. and Dillingham, T. (2008). The incidence of peripheral nerve injury in extremity trauma. Am J Phys Med Rehabil 87(5): 381-385.

19. Wang, W., Degrugillier, L., Tremp, M., Prautsch, K., Sottaz, L., Schaefer, D.J., Madduri, S. and Kalbermatten, D. (2018). Nerve repair with fibrin nerve conduit and modified suture placement. The Anat Rec 301(10): 1690-1696

20. Wilhelm, J. C., Xu, M., Cucoranu, D., Chmielewski, S., Holmes, T., Lau, K. S., Bassell, G. J. and English, A. W. (2012). Cooperative roles of BDNF expression in neurons and Schwann cells are modulated by exercise to facilitate nerve regeneration. $J$ Neurosci 32(14): 5002-5009. 